\author{
О.В. Максимова \\ ФГБОУ ВО «Саратовский государственный медицинский университет им. В.И. Разумовского» МинзАрава России, Саратов, Россия
}

\title{
Аннотация
}

Представлено редкое наблюдение гипотиреоидной комы, развившейся у пациентки с недиагностированным первичным гипотиреозом, осложнившимся развитием рабдомиолиза и Алительного угнетения деятельности Аыхательного центра. Аоминирование в клинической картине заболевания предшествуюшей патологии сердечно-сосудистой системы, выраженного отечного синдрома и гиперхолестеринемии первоначально направи^о диагностический поиск в сторону кардиальной патологии, что замедли^о постановку диагноза гипотиреоза. Цель настоящей публикации - привлечь внимание клиницистов к гипотиреоидной коме как редко встречающемуся осложнению гипотиреоза, протекавшему в данном случае под «маской» ишемической болезни сердца с развитием хронической сердечной недостаточности.

КАючевые слова: гипотиреоз, гипотиреоидная кома, рабдомиолиз

Амя шитирования: Максимова О.В., Чобитько В.Г. Гипотиреоидная кома. Клиническое наблюдение. Терапевтический архив. 2021; 93 (10): 1217-1220. DOI: 10.26442/00403660.2021.10.201080

CASE REPORT

\section{Myxedema coma. Case report}

\author{
Olga V. Maksimova ${ }^{凶}$, Viktoriia G. Chobitko \\ Razumovsky Saratov State Medical University, Saratov, Russia
}

\section{Abstract}

A rare case of hypothyroid coma developed in a patient with diagnosed primary hypothyroidism, complicated by the development of rhabdomyolysis and prolonged oppression of the respiratory center is presented. The predominance of previous cardiovascular pathology, marked oedema syndrome, and hypercholesterolemia in the clinical picture of the disease initially directed the diagnostics of cardiac pathology, which delayed the diagnosis of hypothyroidism. This publication aims to draw clinicians' attention to hypothyroid coma as a rare complication of hypothyroidism, which in this case was "disguised" as ischemic heart disease with the development of chronic heart failure.

Keywords: hypothyroidism, hypothyroid coma, rhabdomyolysis

For citation: Maksimova OV, Chobitko VG. Myxedema coma. Case report. Terapevticheskii Arkhiv (Ter. Arkh.). $2021 ; 93$ (10): 1217-1220. DOI: $10.26442 / 00403660.2021 .10 .201080$

\section{Введение}

Гипотиреоидная кома (ГК), впервые описанная в литературе в 1879 г., представляет собой редкое $(0,22$ случая на 1 млн населения в год) [1], угрожающее жизни осложнение длительно декомпенсированного гипотиреоза. Практически всегда ГК развивается у больных пожилого возраста, преимущественно женщин, с длительно протекающим не диагностированным первичным аутоиммунным гипотиреозом, характеризующимся лимфоидной инфильтрацией с последующим соединительнотканным перерождением тиреоидной ткани, приводящим к снижению ее функции. Так, по данным 800 медицинских центров Германии, средний возраст наблюдавшихся 24 пациентов с ГК составил 73 года [2]. Полиморфизм медленно формирующихся клинических проявлений аутоиммунного гипотиреоза при их малой специфичности затрудняет своевременную постановку диагноза у лиц пожилого возраста, поскольку типичные клинические симптомы гипофункции щитовидной железы (ЩЖ) у данного контингента больных зачастую стерты либо рассматриваются как возрастные изменения, что предрасполагает к поздней диагностике заболевания, а в наиболее тяжелых случаях - развитию ГК [3-5]. Диагностика же первичного гипотиреоза, обусловленного хирургическим вмешательством на ЩЖ или проведенной радиойодтерапии, в силу понятных причин не представляет сложности, поэтому заместительная терапия тиреоидными препаратами в этих случаях назначается вовремя, что минимизирует риск развития ГК. Причиной развития ГК у данных пациентов может быть их низкая комплаентность, приводящая к резкому уменьшению суточной дозы, а чаще - прекращению приема тиреоидных гормонов.

Таким образом, наибольшие трудности при диагностике как гипотиреоза, так и ГК встречаются у пожилых пациентов, ранее не наблюдавшихся по поводу патологии ЩЖ и имеющих разнообразную соматическую патологию, маскирующую симптомы гипотиреоза. Острая декомпенсация длительно существующего нелеченого либо неадекватно леченного гипотиреоза, т.е. ГК, развивается под воздействием различных провоцирующих факторов, к наиболее частым из которых относят присоединение инфекционных и воспалительных процессов, охлаждение, травму, хирургические операции, а также прием сильнодействующих медикаментов (наркотики, снотворные, седативные средства и др.).

Развитие ГК происходит постепенно в течение нескольких недель. Нарастают симптомы гипотиреоза: усиливаются слабость и адинамия, прогрессируют сонливость и заторможенность, переходящие в ступор и даже кому,

\footnotetext{
Информация об авторах / Information about the authors

Максимова Омьга Васимьевна - канд. мед. наук, ассистент кафр. эндокринологии. Тел.: +7(961)640-82-86; e-mail: maksi.53@mail.ru; ORCID: 0000-0002-3294-5717

Чобитько Виктория Григорьевна - кан $\Delta$. ме $\Delta$. наук, $\Delta \mathrm{OL}$. кафр. эн $\Delta \mathrm{O}-$ кринологии. ORCID: 0000-0003-4872-2961
}

Olga V. Maksimova. E-mail: maksi.53@mail.ru; ORCID: 0000-0002-3294-5717

Viktoriia G. Chobitko. ORCID: 0000-0003-4872-2961 
снижается температура тела, наблюдается брадикардия. Замедляется дыхание, нарастают гиперкапния и дыхательный ацидоз, прогрессируют сердечная слабость и артериальная гипотония, что в свою очередь ведет к олигурии, анурии и метаболическому ацидозу [6, 7]. Развивается тяжелая гипоксия мозга, приводящая к нарушению функции жизненно важных центров центральной нервной системы, в результате чего может наступить смерть больного. Тяжесть состояния и жизненный прогноз при ГК в значительной степени определяют нарушения сердечно-сосудистой системы, характеризующиеся развитием гипотиреоидной миокардиодистрофии, приводящей к застойной сердечной недостаточности $[6,8]$. Тяжелое, угрожающее жизни осложнение - тампонада сердца вследствие накопления в полости перикарда большого количества муцинозной жидкости. Еще одним угрожающим жизни осложнением ГК является асептический некроз скелетных мышц - рабдомиолиз, результатом которого может стать острая почечная недостаточность $[6,9]$

Перечисленные тяжелые осложнения в сочетании с трудностями диагностики ГК, встречающимися у пожилых пациентов, ранее не наблюдавшихся по поводу патологии ЩЖ, способствуют позднему началу лечения и приводят к высокой летальности, достигающей в настоящее время $20-50 \%$ случаев [10].

Приводим клиническое наблюдение больной Л. 65 лет, доставленной машиной скорой помощи в ГУЗ ГКБ №8 Саратова. Имелись жалобы на одышку при незначительной физической нагрузке и в покое, усиливающуюся в горизонтальном положении, слабость, сонливость, зябкость, отеки на лице и ногах, увеличившиеся в последний месяц. Сбор анамнеза затруднен из-за выраженной слабости и снижения памяти. По словам сына пациентки, в течение 10 лет у нее отмечаются слабость, снижение работоспособности, отечность лица, изменение тембра голоса (голос стал более низким, грубым), прибавка массы тела на фоне обычного образа жизни и питания. В последние 2 года присоединились отеки нижних конечностей, одышка сначала при небольшой физической нагрузке, а затем и в покое, женщина спала с приподнятым изголовьем, постоянно зябла. Терапевтом диагностирована артериальная гипертензия (АГ). Как сообщено родственником больной, последние 2 нед она постоянно спала, просыпалась только при активных попытках ее разбудить для приема пищи, с трудом вступала в словесный контакт, жаловалась на постоянную зябкость. Врачом скорой помощи, зарегистрировавшим высокий уровень артериального давления (АД) - 200 и 100 мм рт. ст., пациентка доставлена в терапевтический стационар.

При поступлении состояние больной средней тяжести. Она активна в пределах постели. Выраженная сонливость, из-за которой малодоступна словесному контакту. Зрачки $\mathrm{OS}=\mathrm{OD}$, реакция на свет сохранена. Сухожильные рефлексы $\mathrm{D}=\mathrm{S}$, ослаблены. Тонус мышц снижен. Подкожно-жировая клетчатка развита избыточно, распределена равномер-

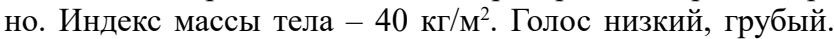
Температура тела $-35,8^{\circ} \mathrm{C}$. Кожа плотная, утолщенная, холодная на ощупь, сухая, на конечностях слущивающийся эпидермис по типу «рыбьей чешуи». Отеки лица, конечностей, передней брюшной стенки. ЩЖ не пальпируется. Волосяной покров на голове редкий. Оволосение в подмышечных областях, лобковой области, нижних конечностях отсутствует. Границы относительной сердечной тупости расширены влево на 1 см кнаружи от срединно-ключичной линии. Тоны сердца глухие, акцент II тона над аортой. Пульс - 64 уд/мин, ритмичный. АД - 130 и 80 мм рт. ст.
В нижних отделах легких ослабленное везикулярное дыхание, притупление перкуторного звука до уровня VII ребра. Частота дыхательных движений - 18 в 1 мин, одышка смешанного характера при небольшой физической нагрузке и в покое. Положение в постели с приподнятым изголовьем. Язык отечный, на боковых поверхностях отпечатки зубов. Живот мягкий, безболезненный при пальпации, притупление перкуторного звука в отлогих частях живота. Печень не пальпируется. У пациентки запоры.

При поступлении в стационар больная осмотрена кардиологом, диагноз: ишемическая болезнь сердца, атеросклеротическая болезнь сердца, АГ 3-й степени, риск 4. Осложнения: хроническая сердечная недостаточность (XCH) 2Б-3 IV функционального класса, двусторонний гидроторакс, асцит, гидроперикард. Назначены внутривенное введение поляризующей смеси объемом 150 мл, мочегонных препаратов, антиагреганты. Через 10 ч пребывания в стационаре произошло дальнейшее угнетение сознания: кома I, АД снизилось до 100/70 мм рт. ст. Пациентка переведена в отделение реанимации, подключена к аппарату искусственной вентиляции легких (ИВЛ).

При проведении электрокардиографического и эхокардиографического исследования не получено данных, позволяющих трактовать имеющийся выраженный отечный синдром только как проявление ХCH. Электрокардиография показала, что синусовый ритм - 82 в 1 мин, горизонтальное положение электрической оси сердца, снижение вольтажа всех зубцов, депрессия зубца $T$. По результатам эхокардиографии отмечены неспецифические дегенеративные изменения атеросклеротического генеза стенок аорты, створок аортального клапана; гипертрофия миокарда левого желудочка; умеренная гипертрофия правого желудочка; фактор выброса 62\%. Также обнаружены недостаточность митрального и трикуспидального клапанов от небольшой до умеренной; легочная гипертензия 1-й степени; жидкость в перикарде объемом 150-200 мл. Наличие компенсации кровообращения по большому кругу не оценивалось (пациентка находилась на ИВЛ). Рентгенография грудной клетки определила, что тень сердца значительно расширена в поперечнике, аорта уплотнена, легочный рисунок усилен. Имеются двусторонний гидроторакс, признаки застоя в системе малого круга кровообращения. По данным ультразвукового исследования органов брюшной полости отмечены увеличение размеров правой доли печени до 182 мм, левой - 86 мм, признаки диффузных изменений паренхимы печени, асцит.

Дальнейший диагностический поиск направили на выяснение причин, вызвавших развитие выраженного отечного синдрома и угнетение сознания. Учитывая жалобы больной и объективные данные, заподозрили длительно существующий декомпенсированный гипотиреоз, возможно, осложнившийся развитием ГК. Данное предположение нашло свое подтверждение в ходе проведенного гормонального обследования, которое выявило наличие первичного гипотиреоза в исходе хронического аутоиммунного тиреоидита: тиреотропный гормон крови - 52 мкМЕ/мл (референсные значения 0,35-4 мкМЕ/мл), свободный тироксин 4,1 пмоль/л (референсные значения 9,0-19,5 пмоль/л), АТ к тиреопероксидазе - 1 тыс. ед. (референсные значения до 30 ед.). Диагноз подтвердили результаты ультразвукового исследования ЩЖ: уменьшение объема до 3,6 мл, признаки аутоиммунного повреждения - пониженная эхогенность, неоднородность структуры.

Наличие декомпенсированного гипотиреоза объясняло также имеющиеся у пациентки тяжелые расстройства липидного обмена (холестерин крови - 11,4 ммоль/л, 
триглицериды - 2,48 ммоль/л, липопротеины низкой плотности - 7,12 ммоль/л, липопротеины высокой плотности - 1,06 ммоль/л) и нормохромную анемию (эритроциты - 2,59 млн, гемоглобин - 78 г/л, цветовой показатель $-0,9)$, поскольку указанные лабораторные изменения являются неотъемлемой составляющей данного заболевания [11], а выраженная дислипидемия, несомненно, способствовала прогрессированию атеросклеротического процесса [12].

Проводимая заместительная терапия левотироксином натрия 200-400 мкг/сут осуществлялась через назогастральный зонд. Пациентка получала глюкокортикоиды, мочегонные, антибактериальные препараты, антиоксиданты, антиагреганты, ноотропы. Осуществлялось парентеральное питание. Через сутки больная пришла в сознание, ориентирована в обстановке, реагирует на окружающие раздражители, понимает обращенную речь. Выраженность отечного синдрома уменьшилась, АД 140/80 мм рт. ст., число сердечных сокращений 68 уд/мин, ритм. По развитию коматозного состояния наладили ИВЛ в режиме гипервентиляции, которая продолжалась в течение 39 сут, поскольку и после восстановления сознания отсутствовала самостоятельная дыхательная деятельность больной.

На фоне применения препаратов тиреоидных гормонов и проведения противоотечной терапии на 40-е сутки восстановилось самостоятельное дыхание, что позволило трактовать имевшееся угнетение дыхательной деятельности как проявление ГК. Другие причины нарушения дыхательной функции, а именно острое нарушение мозгового кровообращения, миастенический криз, органическое поражение головного мозга, нейроинфекция в процессе обследования исключили. По данным компьютерной томографии головного мозга выявлены кистозно-глиозные изменения левого полушария головного мозга в бассейне левой средней мозговой артерии, элементы выраженной субатрофии вещества головного мозга. Электромиография показала снижение скорости проведения нервного импульса по сенсорным и моторным волокнам периферических нервов. Дуплексное сканирование транскраниальных артерий и вен обнаружило признаки легкого спазма средних, передних, задних мозговых артерий, позвоночных артерий, основной артерии. Также признаки умеренной венозной дисциркуляции в базальных, позвоночных венах, большой мозговой вене. Триплексное сканирование магистральных артерий головы на экстракраниальном уровне выявило стеноз: правой общей сонной артерии (СА) в области бифуркации 15-20\% гиперэхогенной атеросклеротической бляшкой; внутренней СА в бульбусе $25-30 \%$ гиперэхогенной атеросклеротической бляшкой; левой общей СА в области бифуркации 15-20\%; левой внутренней СА в устье $30-35 \%$ гиперэхогенной атеросклеротической бляшкой. Признаков нарушения кровотока в артериях не зарегистрировано. Консультация окулиста: гипертоническая ангиопатия сетчатки обоих глаз, поля зрения в норме. Консультация невролога: двигательные функции в конечностях сохранены, мышечная сила $\mathrm{S}=\mathrm{D}$ снижена, рефлекторная активность $\mathrm{S}=\mathrm{D}$ уменьшена, патологических знаков, менингиальных симптомов нет, кистевая миастеническая проба отрицательная.

Таким образом, результаты проведенного клинико-инструментального обследования не выявили органических поражений центральной и периферической нервной системы.

Внимание также привлекали изменения в биохимическом анализе крови, а именно повышенный уровень мо- чевины (10,96 ммоль/л) и креатинина (300 ммоль/л), что в сочетании с обнаруженным повышенным уровнем креатининфосфокиназы - 707 ед/л (референсные значения 25-175 ед/л), креатининкиназы - 240 ед/л (референсные значения 0-190 ед/л) и щелочной фосфатазы - 254,5 ед/л (референсные значения 0-177 ед/л) позволило заподозрить асептический некроз скелетных мышц с развитием рабдомиолиза. Последний, как известно, может встречаться при ГК, приводить к накоплению азотистых соединений в крови и формированию почечной недостаточности, что и зарегистрировали у пациентки. Согласно литературным данным [6], рабдомиолиз способствует развитию метаболического ацидоза, гиповентиляции альвеол, гиперкапнии, гипоксии тканей, в том числе головного мозга, приводящих к угнетению деятельности дыхательного центра. Дополнительными, усугубляющими гипоксию факторами у больной, по всей вероятности, являлись выраженное ожирение, длительная неконтролируемая АГ, $\mathrm{CH}$, плевральный выпот, а также свойственные декомпенсированному гипотиреозу отечность слизистых оболочек дыхательных путей. На фоне проведенной заместительной терапии гипотиреоза перечисленные лабораторные показатели нормализовались.

После восстановления самостоятельной дыхательной деятельности пациентку перевили в общее отделение. За время пребывания в стационаре на фоне проводимой терапии практически исчезли отеки, восстановилось самостоятельное дыхание, гемодинамические показатели, больная активна в пределах палаты, нормализовался уровень свободного тироксина в крови (14,0 пмоль/л), значительно уменьшился уровень тиреотропного гормона (12 мкМЕ/мл).

Пациентку выписали на амбулаторное лечение с диагнозом: первичный гипотиреоз тяжелой формы в исходе хронического аутоиммунного тиреодита, стадия длительной декомпенсации; ишемическая болезнь сердца, атеросклероз аорты, коронарных, сонных артерий, АГ 3-й степени, риск 4. Дислипидемия. Осложнения. ГК. Дыхательная недостаточность 3-й степени. Угнетение дыхательного центра, ИВЛ, трахеостомия. Дисметаболическая миокардиодистрофия гипотиреоидного, гипертонического генеза. ХCH 2Б-3 IV функционального класса. Гидроперикард, двусторонний гидроторакс, асцит. Хроническая ишемия головного мозга смешанного генеза. Рабдомиолиз гипотиреоидного генеза. Транзиторное нарушение функции почек. Анемия средней степени. Сопутствующие заболевания. Экзогенно-конституциональное ожирение 3-й степени.

\section{Заключение}

Таким образом, наличие у пациентки своевременно не диагностированного первичного гипотиреоза, сформировавшегося в исходе хронического аутоиммунного тиреоидита, привело к развитию тяжелого, угрожающего жизни осложнения - ГК с угнетением дыхательного центра, дисметаболической миокардиопатией и почечной недостаточностью, обусловленной рабдомиолизом. Доминирование в клинической картине у пациентки с предшествующей патологией сердечно-сосудистой системы выраженного отечного синдрома первоначально направило диагностический поиск в сторону кардиальной патологии, что замедлило постановку диагноза гипотиреоза. Дополнительных факторов, спровоцировавших у пациентки ГК, выявить не удалось. Скринингу на гипотиреоз в первую очередь подлежат больные старших возрастных групп с отечным синдромом, дислипидемией. Своевременная диагностика и регулярно проводимая заместительная терапия гипотиреоза 
профилактируют развитие ГК. Цель настоящей публикации - привлечь внимание клиницистов к ГК как редко встречающемуся, однако сопровождающемуся высокой летальностью осложнению гипотиреоза.
Конфликт интересов. Авторы заявляют об отсутствии конфликта интересов.

Conflict of interests. The authors declare no conflict of interest.

\section{Список сокрашений}

АГ - артериальная гипертензия

АД - артериальное давление

ГК - гипотиреоидная кома

ИВЛ - искусственная вентиляция легких
СА - сонная артерия

$\mathrm{XCH}$ - хроническая сердечная недостаточность

ЩЖ - щитовидная железа

\section{АИТЕPATYPA/REFERENCES}

1. Петунина Н.А. Гипотиреоидная кома. Эндокринология. Национальное руководство. М.: ГЭОТАР-Медиа, 2018; с. 528-33 [Petunina NA. Myxedema coma. Endocrinology. National guidelines. Moscow: GEOTAR-Media, 2018; p. 528-33 (in Russian)].

2. Петунина Н.А. Гипотиреоидная кома. Эндокринология. Национальное руководство. М.: ГЭОТАР-Медиа, 2012; с. 568-74 [Petunina NA. Myxedema coma. Endocrinology. National guidelines. Moscow: GEOTAR-Media, 2012; p. 568-74 (in Russian)].

3. Мкрутумян А.М., Нелаева А.А. Неотложная эндокринология М.: ГЭОТАР-Медиа, 2018 [Mkrtumyan AM, Nelaeva AА. Emergency endocrinology. Moscow: GEOTAR-Media, 2018 (in Russian)].

4. Sethi PI, Kamal MI, Verma SI, Bhatia P. Undiagnosed myxedema coma: Rare but life-threatening. Saudi J Anaesth. 2018;12(1):173-74. DOI:10.4103/1658-354x.222365

5. Milkau M, Sayk F. Thyreotoxische Krise und Myxödemkoma. DMW - Deutsche Medizinische Wochenschrift. 2018;143(06):397-405. DOI:10.1055/s-0043-111728

6. Лукьянчиков В.С. Эндокринные комы и кризы. М.: Академический Проект, 2013 [Lukyanchikov VS. Endocrine coma and crises. Moscow: Publishing House Academic Project, 2013 (in Russian)].

7. Orlova M, Rodionova T. Functional state of kidneys in patients with clinical manifestations of hypothyroidism. Russian Open Medical Journal. 2013;2(2):0204. DOI:10.15275/rusomj.2013.0204

8. Трошина Е.А., Юкина М.Ю., Огнева Н.А., Мазурина Н.В Нарушения функции щитовидной железы и сердечно-сосудистая система. Клиническая и экспериментальная тиреоидология. 2010;6(1):12 [Troshina EA, Yukina MIu, Ogneva NA,
Mazurina NV. Thyroid function disorders and cardiovascular system. Clinical and experimental thyroidology. 2010;(6)1:12 (in Russian)]. DOI:10.14341/ket20106112-19

9. Петров А.В., Луговая Л.А., Стронгин Л.Г., Некрасова Т.А. Недиагностированный гипотиреоз - фактор риска возникновения рабдомиолиза при терапии статинами. Клиническая $u$ экспериментальная тиреоидология. 2014;10(4):26-33 [Petrov AV, Lugovaya LA, Strongin LG, Nekrasova TA. Undiagosed hypothyroidism fs risk factor of statin-induced rhabdomyolysis Clinical and experimental thyroidology. 2015;10(4):26-33 (in Russian)]. DOI: $10.14341 /$ ket2014426-33

10. Ono Y, Ono S, Yasunaga H, et al. Clinical characteristics and outcomes of myxedema coma: Analysis of a national inpatient database in Japan. J Epidemiol. 2017;27(3):117-22. DOI:10.1016/j.je.2016.04.002

11. Долбин И.В., Екимовских А.Ю. Гипотиреоз и ишемическая болезнь сердца: современное состояние проблемы. Медицинский Альманах. 2016;4(44):121-6 [Dolbin IV, Ekimovskikh AIu. Hypothyroidism and ischemic cardiac disease: modern condition of the issue. Meditsinskii Almanakh. 2016;4(44):121-6 (in Russian)].

12. Петунина Н.А., Трухина Л.В., Мартиросян Н.С. Дисфункция щитовидной железы и система кроветворения. Клиническая и экспериментальная тиреодология. 2011;7(4):27-31 [Petunina NA Truhina LV, Martirosian NS, Thyroid dysfunction and hematopoietic system. Clinical and experimental thyroidology. 2011;7(4):27-31 (in Russian)]. DOI:10.14341/ket2011742

Статья поступила в редакцию / The article received: 30.03.2019

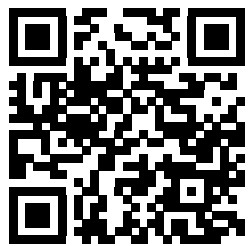

OMNIDOCTOR.RU 